\title{
Comparison of Nusinersen and Evrysdi in the Treatment of Spinal Muscular Atrophy
}

\author{
Xiaoying $\mathrm{Zhu}^{1, *}$ \\ ${ }^{1}$ USC Master graduate, School of Pharmacy, Healthcare decision and analysis, USA
}

\begin{abstract}
Spinal Muscular Atrophy (SMA) is a genetic neuromuscular disease that commonly affects children, and usually worsens with age that often leads to permanent disability and death for many of the SMA patients. Recently, two drugs are developed to improving the quality of life of SMA sufferers: Evrysdi and Nusinersen. This study is identified by a systematic literature review to compare two treatments. The comparison attempts to focus on mechanism, administration and clinical trials. The trials include the ENDEAR study for Nusinersen, and the FIREFISH study for Evrysdi. Due to the different baselines of two trials, matching-adjusted indirect comparison (MAIC) is used to "weighted" baseline characteristics to match each other across all the studies. Each of the trials highlighted the effectiveness for comparison. Both Nusinersen and Evrysdi have had a major and positive impact on improving the quality of life of SMA, and both therapies have been shown to be highly effective. Moreover, the indirect comparison with Matching Adjustment Indirect Comparison shows that Risdiplam is more effective as compared to Nusinersen. Nonetheless, the comparison is still inaccurate due to lack of real-world evidence from patients.
\end{abstract}

\section{Introduction}

Spinal Muscular Atrophy is a genetic neuromuscular disease that often leads to permanent disability and death for many of the sufferers. It is caused by the loss of specialized nerve cells, called motor neurons that are the nerves that muscle movement. On a genetic level, this occurs as a result of mutations that occur in the SMN1 gene, that lead to the disruption or end of production of the survival motor neuron (SMN) protein structures that are essential for the building of muscle [1]. As a result, the nerves slowly break down in both the brain and spinal cord, leading to gradual weakening of nerve impulse transfer between the brain and the muscles. This weakness has been found to affect proximal muscles or muscles close to the center of the body, compared to distal muscles or muscles located away from the center of the body.

The disease commonly affects children, and usually worsens with age. As such, the symptoms often begin with the shrinking of muscles and difficulty in controlling movements that are usually easy to do such as sitting up, and moving head. As symptoms progress, the child may be unable to work properly and may eventually lose the use of their legs [1]. Many sufferers often end up having difficulty swallowing and breathing as symptoms progress. SMA has an estimated incidence range from 1 in 6,000 to 1 in 10,000 live births, and frequency of carrier range from $1 / 40$ to $1 / 60$ [1]. Since there are no head-to-head studies for these two treatments, it is hard to make a strict comparison. The only data could be collected for comparisons is effectiveness from clinical trials [2]. Therefore, this study is identified by a systematic literature review to compare two treatments.

\section{The phenotype of Spinal Muscular Atrophy}

There are several types of Spinal Muscular Atrophy. Type 1 is the most severe and has the highest mortality rate [1]. This type of SMA develops in the fetus during pregnancy and is often identified by fewer womb movements during pregnancy. When the child is born, they usually have joint problems, weak muscles as well as problems breathing. The inability to breath properly is usually the cause of the high mortality. It also usually emerges shortly after birth. The child may not be able to hold up their head, may have floppy arms and legs due to lack of control, as well as problems swallowing [1]. Because of the issue of breathing, many of the children with type 1 SMA do not live past the age of two.

SMA Type 2 is often moderate rather than severe and leads to the child being disabled throughout their lives. It usually emerges 7 to 18 months after birth and is characterized by the loss of the child's legs [1]. In some cases, the child may also lose part or all of their movement of the arms. But in most moderate cases, the child may be able to walk and sit with some help.

Although SMA is not curable, it can be managed through medication that prolongs the life of the child, especially if the child has Type 2 SMA as well as early-stage Type 1 SMA.

*Corresponding author: xzhu4711@usc.edu 


\section{The therapy of Spinal Muscular Atrophy}

Evrysdi or Risdiplam and Nusinersen also known as Spinraza are two drugs recently developed to improving the quality of life of SMA sufferers. Both drugs have proved to be highly effective in the treatment of SMA. Both drugs rely on a natural phenomenon that was discovered to be occurring in the body.

Humans have a "backup" SMN gene, known as SMN2 [3]. This gene undergoes slight differences in its sequence known as "alternative splicing" which leads to genetic distortion that restricts the amount of functional SMN it produces to around 10 to $15 \%$. Alternative splicing leads to the production of several types of different proteins. It has a phenomenon when it comes to changes in the messenger RNA or mRNA leads to changes in the final protein. In the nerve, exon splicing relies on a delicate balance between splicing enhancers, that is, DNA sequences that take on proteins to stimulate exon inclusion and splicing silencers, those that take on splicing suppressor proteins to which prevent the process of exon inclusion in the final mRNA sequence [3]. As such, a slight change in SMN2's gene sequence leads to distortions in the exon due to the imbalance between exon 7 splicing enhancers and silencers. Due to this distortion, the exon is excluded from most of the SMN2 mRNA molecules, leading SMN2 proteins that are shorter and fail to properly function.

\section{The comparison of two treatments: Nusinersen versus Evrysdi}

Nusinersen is a drug that was approved in 2016 to manage the symptoms of SMA. It is highly effective, and has been shown to have significant benefits to those with SMA. It is usually administered as an injection. The injection comes as a solution that introduce into the body intrathecally, that is, through the fluid-filled space of the spinal canal [4]. Because the fluid filled space has access to the brain, the medication is able to cross the blood-brain barrier and thus have an effect on the brain. Due to the difficulty in injecting into the spine, as well as the sensitivity are to be injected, the injection is administered only by a doctor in a medical office or clinic. The dosage regimen starts with 4 initial doses, that is once every 2 weeks for the first 3 doses and again 30 days after the third dose and then is given once every 4 months thereafter.

Nusinersen is an Antisense oligonucleotide drug. Antisense oligonucleotide drugs (ASOs) are drugs designed to introduce synthetic genetic material in small quantities that bind to ribonucleic acid (RNA). Such synthetic genetic material is specifically designed to target particular genes and modify how that particular gene is read [4]. As a result, ASOs have several applications when it comes to SMA, as the drugs can target the SMN2 gene with the aim of converting it to be read as an SMN1 gene, that is, the genetic material introduced "patches up" the SMN2 gene and makes it mimic the actions and functions of the SMN1 gene [5]. As such, ASOs like Nusinersen fix the splicing errors in genes such as SMN2. This allows for the drug to effectively slow down both SMA type 1 and type 2, especially if the drug is administered before a large number of nerves have been damaged [5]. The fact that it should be administered only at various intervals of four months, also makes it quite easy for patients to handle without forgetting to take their medication on time. As a result, the medication has been rated highly.

Evrysdi is also an (SMN2) mRNA splicing modifier. However, unlike Nusinersen, it is administered orally, rather than through an injection and is the first and only medicine for SMA that sufferers can take on their own and at home, without supervision from a doctor. Evrysdi aims to increase and sustain the SMN protein levels to maintain nerves in both the central nervous system (CNS) as well as peripheral tissues which are usually the first to be damaged by SMA. The reason why Evrysdi can be taken through oral administration is because it is made of a very small molecule that has been designed and optimized to be as safe and as highly targeted as possible [6]. The small molecule size allows it to cross the blood-brain barrier, and allows for easy uptake by peripheral tissues. This is because Evrysdi's molecular weight is approximately 400 Dalton which is small enough to cross the blood-brain barrier. On the other hand, Nusinersen has a molecular weight of more than 7,000 Dalton, which is why it cannot be taken orally.

Table 1. Common information comparison

\begin{tabular}{|l|l|l|}
\hline $\begin{array}{l}\text { Comparison } \\
\text { Items }\end{array}$ & Nusinersen (Spinraza) & $\begin{array}{l}\text { Evrysdi } \\
\text { (Risdiplam) }\end{array}$ \\
\hline $\begin{array}{l}\text { First approval } \\
\text { date }\end{array}$ & December 2016 & August 2020 \\
\hline Company & Biogen & Genentech \\
\hline Dosage & Injection & Oral \\
\hline Drug type & $\begin{array}{l}\text { An antisense } \\
\text { oligonucleotide }\end{array}$ & Small molecule \\
\hline $\begin{array}{l}\text { The most } \\
\text { common } \\
\text { adverse } \\
\text { effects }\end{array}$ & $\begin{array}{l}\text { Constipation, lower } \\
\text { respiratory infection, } \\
\text { pyrexia, headache, } \\
\text { vomiting and back } \\
\text { pain. }\end{array}$ & $\begin{array}{l}\text { Fever, diarrhea, } \\
\text { rash, upper } \\
\text { respiratory tract } \\
\text { infections, } \\
\text { pneumonia, } \\
\text { constipation, } \\
\text { vomiting. }\end{array}$ \\
\hline
\end{tabular}

When making a comparison of the two medications, as highlighted earlier Biogen's Nusinersen was approved earlier, in late 2016 as the first therapy available for SMA sufferers. Nusinersen is available to all SMA type patients without restrictions. On the other hand, Evrysdi, which is marketed by Genentech, a subsidiary of Roche is currently approved for patients who are 2 months of age and older with all types of SMA [6].

The two drugs went through different trials, mostly focused on even infants, up to 6 or 7 months old, with the more severe SMA type 1 disease. This is because type 1 is the most common type of SMA and it emerges 
shortly after birth. The trials include the ENDEAR study for Nusinersen, and the FIREFISH study for Evrysdi [6]. Each of the trials highlighted the effectiveness of both types of medication in the treatment of SMA Type 1 and thus both medications are recommended and it is up to the discretion of the doctor. However, making direct comparisons makes it problematic for several reasons.

To date, no comparative studies of both drugs has been done. As such, most of the data on the effectiveness of both therapies has come from the earlier studies highlighted and from clinical trials. It is important to highlight that the studies were done at different times, with different sample populations [2]. However, one can look at a number of factors that may make it possible to make some comparisons. A preliminary indirect treatment comparison has focused on the FIREFISH study and ENDEAR study [7]. Given that the studies use different baselines, Matching-adjusted indirect comparison (MAIC) is done, whereby baseline characteristics are 'weighted' and adjusted so that the summary baseline characteristics match each other across all the studies [7]. These baseline characteristics include the SMN2 copy number whereby all the patients had two copies of the gene, duration of symptoms, age at time of first dose, functional score at baseline and ventilatory/nutritional support. This is because these factors have been shown to have a major influence on the outcome of the treatment. Risdiplam has been shown to be highly effective when it comes to the treatment of Types 1, 2 and 3 SMA. It has been shown to stop many of the debilitating effects of the disease. Nusinersen also had good outcomes and overall, both drugs have been found to be highly effective.

Nonetheless, a comparison between the two treatments both when using Matching Adjustment Indirect Comparison or unadjusted data shows that Risdiplam is more effective as compared to Nusinersen, especially when it comes to infant onset SMA. Overall, it was established that Risdiplam leads to higher survivability rates and had better performance on motor milestone response of the infants of over 85 percent if the treatment is administered early. As such, it is a treatment that gets a higher recommendation as compared to Nusinersen.

\section{Discussion}

It has to look at the weaknesses of the MAIC method of comparison, whereby biases may emerge if not all prognostic and predictive factors are included. The method also does not factor in the potential changes in standard of care that often occur over time. The process of reducing sample sizes in the re-weighting process also increases uncertainty in the results, and reducing accuracy [7].

At the time of the ENDEAR study for Nusinersen, although there was not much information on how much care infants with SMA type 1 were not aware of the degree of help that could be given to infants with SMA, especially SMA Type 1 [8], but given that Nusinersen does not entail having to take medication every day and comes with the advantage of the child being able to minimize the number of hospital visits, as well as the parent not having to remember to give the child medication on a daily basis. Nonetheless, Nusinersen is usually not an easy option for those with scoliosis and other diseases that affect the spine.

Evrysdi does has several advantages when looks at its features. Given its relatively recent approval, it has so far shown to have an excellent safety profile. Of the two drugs, it is also the drug that has been shown to be effective in the broadest range of SMA patients in terms of type, age and severity. This is due to the sample population that was involved in the second and third FIREFISHs study had patients ranging from ages 1 month to 25 years with the three types of SMA [6]. As such, of the two studies that focused on the two medications, Evrysdi had a more extensive sample population. The drug has been shown to not only significant improvements in the younger patients, but also those who are older saw their SMA stabilize or improve, leading to a significant improvement in their quality of life. Another major advantage of Evrysdi is that it targets every cell in the body. Recent research has been indicating that SMA is a body-wide disease. On the other hand, Nusinersen mainly targets motor neurons as they are usually the nerve cells most affected by SMA [8].

While some patients may find Evrysdi's daily oral administration a bit of a challenge as compared to the Nusinersen's injection of once every four months, many doctors as well as many patients may find that oral administration poses a smaller burden as compared to Nusinersen. It is easy for doctors to ship the syrup to patients from the doctor and given that the medicine is flavored, many patients find it easy to take on a daily basis. In the current context of the COVID-19, those taking Evrysdi have had the advantage of avoiding congested hospitals where the risk of COVID-19 transmission is high, as well as giving doctors the opportunity to focus on battling the pandemic [2].

\section{Conclusion}

In conclusion, both Nusinersen and Evrysdi have had a major and positive impact on improving the quality of life of SMA sufferers. Both therapies have been shown to be highly effective, although the indirect comparison with Matching Adjustment Indirect Comparison shows that Risdiplam is more effective as compared to Nusinersen. However, Nusinersen is more suitable for those who do not mind having injections into their spine and find it more convenient to get the injections three times a year after the initial two injections. Unlike Nusinersen, Evrysdi is more convenient for those who prefer to take the medication orally which also saves on consultation fees with doctors. As such, despite the different routes of administering the therapies, they work in a similar manner, patients can choose either two depending on which one is considered more convenient as well as depending on the advice from the doctor. 
Given that so far in the past two years, there has been no safety issues with Evrysdi, which is why it is approved in the United States. Currently, the therapy is yet to be approved in the European Union, thus patients in Europe only have access to Nusinersen.

Further study will focus on the real-world evidence of the studies to make strict comparisons, SMA treatments comparison is still inaccurate until more data collected from patients across different areas, ages, types, the severity of the disease, and genetic information.

\section{Acknowledgement}

The author is grateful to Dr. Liu for helpful guidance of the paper.

\section{References}

1. Kolb, S. J., Kissel, J. T. Spinal muscular atrophy. Neurologic clinics, 33(4), 831-846 (2015).

2. Figueiredo, M. Comparing SMA Therapies Neither Easy Nor Fair, Clinicians Say. Retrieved January 07, 2021, from

https://smanewstoday.com/news-posts/2020/08/21/c omparing-sma-therapies -neither-easy-nor-fair-clinicians-say/ (2020).

3. Scoto, M., Finkel, R. S., Mercuri, E., \& Muntoni, F. Therapeutic approaches for spinal muscular atrophy (SMA). Gene therapy, 24(9), 514-519 (2017).
4. Gidaro, T., Servais, L. Nusinersen treatment of spinal muscular atrophy: current knowledge and existing gaps. Developmental Medicine \& Child Neurology, 61(1), 19-24 (2019).

5. Corey, D. R. Nusinersen, an antisense oligonucleotide drug for spinal muscular atrophy. Nature neuroscience, 20(4), 497-499 (2017).

6. La Roche, H. Investigate Safety, Tolerability, PK, PD and Efficacy of Risdiplam (RO7034067) in Infants With Type1 Spinal Muscular Atrophy - Full Text View. Retrieved January 07, 2021, from https:/clinicaltrials.gov/ct2/show/NCT02913482 (2016)

7. Daigl, M., Kotzeva, A., Gorni, K., Evans, R., Hawkins, N., Scott, D., . . . Servais, L. Pro12 How Does Risdiplam Compare In Infantile Onset Spinal Muscolar Atrophy (Sma)? Preliminary Indirect Treatment Comparisons Based On Firefish Part 1 Data. Value in Health, 22. doi: 10.1016/j.jval.2019.09.2344 (2019)..

8. Dangouloff, T., Servais, L. Clinical Evidence Supporting Early Treatment Of Patients With Spinal Muscular Atrophy: Current Perspectives. Therapeutics and Clinical Risk Management, Volume 15, 1153-1161. doi:10.2147/tcrm.s172291 (2019). 